Copyright (C 2014 IEEE. Personal use of this material is permitted. However, permission to reprint/republish this material for advertising or promotional purposes or for creating new collective works for resale or redistribution to servers or lists, or to reuse any copyrighted component of this work in other works must be obtained from the IEEE.

This material is presented to ensure timely dissemination of scholarly and technical work. Copyright and all rights therein are retained by authors or by other copyright holders. All persons copying this information are expected to adhere to the terms and constraints invoked by each author's copyright. In most cases, these works may not be reposted without the explicit permission of the copyright holder.

Baha A. Alsaify, Dale R. Thompson, and Jia Di, "Exploiting hidden Markov Models in identifying passive UHF RFID tags," in Proc. IEEE Radio and Wireless Symposium (RWS), Newport Beach, California, Jan. 19-22, 2014, pp. 259-261. 


\title{
Exploiting Hidden Markov Models in Identifying Passive UHF RFID Tags
}

\author{
Baha' A. Alsaify ${ }^{1}$, Dale R. Thompson ${ }^{2}$, and Jia $\mathrm{Di}^{2}$ \\ ${ }^{1}$ Hijjawi Faculty for Engineering Technology, Yarmouk University, Irbid, Jordan 21163. \\ ${ }^{2}$ Computer Science and Computer Engineering Department, University of Arkansas, \\ Fayetteville, Arksansas 72701.
}

\begin{abstract}
In this work, we identify RIFD tags using hidden Markov models. By isolating the tag's transmission, we differentiate tags by their acquired transmissions. The results acquired from experimenting with hidden Markov models show that the system's performance will improve when different observations are combined together. The proposed system yielded an accuracy of $97.67 \%$ when identifying a tag's manufacturer and an accuracy of $98.04 \%$ when identifying individual tags.

Index Terms-RFID, Hidden Markov Models, Identification, Passive UHF Tags.
\end{abstract}

\section{INTRODUCTION}

Identification refers to the process in which an object can be uniquely recognized. In this work, we are focusing on the problem of identifying a passive ultra-high frequency (UHF) radio frequency identification (RFID) tag. One of the main challenges of RFID technology is power. Passive tags do not have a dedicated power source to supply it with the necessary power to operate; it relies on the received radio frequency (RF) transmission from the RFID reader. Through a process called harvesting, the passive RFID tag will get the energy it needs to activate and communicate with the reader. The harvesting process provides the tag with a very limited power supply which limits the scope of applications we can use over RFID infrastructure. Applications such as cryptography, or complex security algorithms are out of the question. In this paper, we aim to develop and RFID identification system that can work under all of the RFID limitations.

The rest of this paper is organized as follows. In Section II, an overview of some of the work that had been done in the area of identifying RFID tags is presented. Section III provides a closer look on the performed experiments and their setup, followed by the experiments results in Section IV. Finally, the paper's conclusion is presented in Section V.

\section{RELATED WORK}

Identifying objects using their physical characteristics is not a new idea. There are many techniques that can be used to identify objects using their fingerprints. One of the techniques that can be used to identify objects based on their behaviour is the hidden Markov model (HMM) [1]. In this work, we focus on distinguishing between different RFID tags by building the appropriate HMM models. Other techniques can be used to identify RFID tags. In [2], Senthil et al. exploits the tag's minimum power response at multiple frequencies to fingerprint passive UHF RFID tags. The minimum power response was measured using a Voyantic Tagformance Lite system [3] in an anechoic chamber. Another technique to identify RFID tags is introduced in [4]. In this work, the researchers were fingerprint RFID tags by adding a random scattering structure to the tag. In the performed experiments, the distance between the reader and the tag was very close, between $1 \mathrm{~mm}$ and $8 \mathrm{~mm}$. The authors measure the nearfield response of the added structure when creating the fingerprint. A misalignment noise in the readings exists because of mounting issues. Identifying and fingerprinting RFID tags can be also done by extracting several features from the received tag's transmission as shown in [5]. In [5], the work's focus is on extracting timing and power features. A special-built RFID reader is used to challenge the tags by initiating an inventory process. Between the performed experiments, the tag is powered down and is assumed to have lost all of its stored energy before being activated again for the next experiment.

A comparison with the previously mentioned related work is presented in Table I.

\section{EXPERIMENT SETUP}

As mentioned before, the purpose of this work is to build a system using HMM that distinguish between RFID tags. A programmable reader from [6] was used to gather the features we needed. All of the experiments were done in a noisy environment. The distance between the reader's antenna and each of the tags was fixed to be $20 \mathrm{~cm}$ and a population of 300 tags from three different manufacturers was tested. After each experiment run, the tag is assumed to have lost all of its stored energy. In order for HMM to work properly, it needs to be provided with a stream of data that represent the observations gathered from 
TABLE I: Comparison with related work

\begin{tabular}{|c|c|c|c|c|}
\hline & Freq. & Environment & \# Tags & Performance \\
\hline Proposed & UHF & uncontrolled & 300 & $\begin{array}{c}\text { Voltage: average TPR }=79.00 \% \text {; Average accuracvy }=86.00 \% \\
\text { Power: average TPR }=91.33 \% \text {; Average accuracy }=94.22 \% \\
\text { Timing: average TPR }=89.33 \% \text {; Average accuracy }=92.89 \% \\
\text { Timing and power, same model: Average TPR }=58.00 \% \text {; } \\
\text { Average accuracy }=72.00 \% \\
\text { Timing and power, separate models: Average TPR }=94.67 \% \text {; } \\
\text { Average accuracy }=96.45 \%\end{array}$ \\
\hline Periaswamy et al. 2010 [2] & $\mathrm{UHF}$ & controlled & 100 & $\begin{array}{c}\text { Manufacturer-1: TPR }=94.4 \% \\
\text { Manufacturer-2: TPR }=90.7 \% \\
\text { Both Manufacturers: TPR }=90.5 \%\end{array}$ \\
\hline Zanetti et al. 2010 [5] & $\mathrm{UHF}$ & $\begin{array}{l}\text { uncontrolled } \\
\text { controlled }\end{array}$ & 70 & $\begin{array}{l}\text { Tag Identification: } \text { Accuracy }=71.4 \% \text {; using timing features } \\
\text { Tag Identification: Accuracy }=43.2 \% \text {; using power features } \\
\text { Tag Identification: Accuracy }=98.7 \% \text {; using power and timing features } \\
\text { Tag Identification: Accuracy }=99.6 \% \text {; using spectral features }\end{array}$ \\
\hline Lakafosis et al. 2011 [4] & $\begin{array}{l}\mathrm{UHF} \\
(8 \mathrm{~mm})\end{array}$ & controlled & 1 (multiple CoA) & $\begin{array}{l}\text { Same CoA Error Probability: } 10^{-200} \\
\text { All CoA's Error Probability: } 10^{-300}\end{array}$ \\
\hline
\end{tabular}

monitoring the tag's transmission. The observation stream we used to identify tags is the time-voltage waveform of the tag's PC+EPC+CRC transmission. In this work, we are identifying tags based on their manufacturer and based on their individual entities. For each identification problem a total of five experiments were conducted. In the first experiment, we used the raw data we gathered as the observation stream. In the remaining four experiments, we processed the time-voltage wave form. All the HMM experiments were conducted using the Matlab toolbox [7] which was designed based on the theoretical knowledge presented in [1].

\section{RESUlts}

In this section, we present the results of the experiments we conducted. Within this section, there are two main topics. First we perform the tag's manufacturer identification. In the second subsection, we perform the individual tag identification.

\section{A. Tag Manufacturer Identification}

In the tag's manufacturer identification experiments we performed, the true positive rate (TPR), accuracy, and the aria under curve (AUC) for each of the experiments are listed.

1) Voltage Observations: In this experiment, the timevoltage waveform is recorded and used to generate the HMM models. No processing was involved in this experiment. Table II shows the results of this experiment. We determined that tags from manufacturer $\mathrm{B}$ have the best TPR with $87 \%$, while tags from manufacturer $\mathrm{C}$ have the best accuracy and AUC with $92.67 \%$ and $96.16 \%$, respectively.

2) Power Observations: In this experiment, we processed the raw time-voltage observations by dividing the data we have into frames, each of them has ten observations. We then calculated the power contained in each frame. To determine how much improvement did we get from processing the observations, we gathered the TPR, accuracy, and AUC. These measurements and provided in Table II.

The results from this experiment show that preprocessing the data will result in better performance. We expect that further preprocessing will improve the results even more. We also expect that combining different data types will yield better performance as the case with classical pattern recognition algorithms.

3) Timing Observations: In this experiment, we use the transition points between high and low voltages in the gathered time-voltage waveform. We calculate the time between each of the points, and use the intervals as the observations input for the HMM. As before, we calculated the TPR, accuracy, and AUC metrics for this experiment. These metrics are provided in Table II.

As Table II shows, tags from manufacturer $\mathrm{C}$ has the lowest AUC, which was a surprise since they had better AUC when raw voltages or power is used as observations.

4) Combining power and time in the same model: In this experiment, we combine both the power and timing observations. To combine observations, we first divided the voltage observation stream into frames as we did in the third experiment. Each frame sequence is translated into two observations; time and power. After we built the necessary models, we followed the same procedure as before. The TPR, accuracy, and AUC shown in Table II.

5) Combining the results of the time and power models: In this experiment we decided to again combine the power and time observations, but instead of using the power and time observations to build a single Markov model, we are building a power model and a timing model and then we are combining the results of the models based on a weighting factor. We use trial and error to determine the best weight matrix. The TPR, accuracy, and AUC are provided in Table II.

\section{B. Individual Tag Identification}

The second set of experiments that were conducted is similar to the first set in the essence that five experiments 
TABLE II: Tag Manufacturer Identification Results

\begin{tabular}{|c|c|c|c|}
\hline \multicolumn{4}{|c|}{ Voltage Observations } \\
\hline Manufacturer & TPR & Accuracy & $\mathbf{A U C}$ \\
\hline$\overline{\mathbf{A}}$ & $68.00 \%$ & $79.33 \%$ & $79.38 \%$ \\
\hline B & $87.00 \%$ & $86.00 \%$ & $94.87 \%$ \\
\hline $\mathbf{C}$ & $82.00 \%$ & $92.67 \%$ & $96.16 \%$ \\
\hline \multicolumn{4}{|c|}{ Power Observations } \\
\hline Manufacturer & TPR & Accuracy & $\mathbf{A U C}$ \\
\hline$\overline{\mathbf{A}}$ & $86.00 \%$ & $92.00 \%$ & $93.89 \%$ \\
\hline B & $93.00 \%$ & $93.67 \%$ & $93.78 \%$ \\
\hline C & $95.00 \%$ & $97.00 \%$ & $97.50 \%$ \\
\hline \multicolumn{4}{|c|}{ Time Observations } \\
\hline Manufacturer & TPR & Accuracy & $\mathbf{A U C}$ \\
\hline$\overline{\mathbf{A}}$ & $91.00 \%$ & $90.67 \%$ & $97.09 \%$ \\
\hline B & $94.00 \%$ & $97.33 \%$ & $75.29 \%$ \\
\hline $\mathbf{C}$ & $83.00 \%$ & $90.67 \%$ & $37.18 \%$ \\
\hline \multicolumn{4}{|c|}{ Power and Time in the Same Model } \\
\hline Manufacturer & TPR & Accuracy & $\overline{\mathbf{A U C}}$ \\
\hline $\mathbf{A}$ & $82.00 \%$ & $67.33 \%$ & $74.87 \%$ \\
\hline B & $42.00 \%$ & $69.33 \%$ & $60.10 \%$ \\
\hline $\mathbf{C}$ & $50.00 \%$ & $79.33 \%$ & $69.49 \%$ \\
\hline \multicolumn{4}{|c|}{ Power and Time in Separate Models } \\
\hline Manufacturer & TPR & Accuracy & $\overline{\mathbf{A U C}}$ \\
\hline $\mathbf{A}$ & $91.00 \%$ & $97.00 \%$ & $45.37 \%$ \\
\hline B & $97.00 \%$ & $97.67 \%$ & $83.42 \%$ \\
\hline $\mathbf{C}$ & $96.00 \%$ & $94.67 \%$ & $97.18 \%$ \\
\hline
\end{tabular}

were performed by varying the observation stream. The difference between the first experiment set and the second set lies in what is being identified. In the first set, the tag's manufacturer was being identified, while in the second set the individual tag is being identified. In the data collection phase of this work, we tested 100 tags of each manufacturer. Each tag was tested five times. The results for the individual tag identification are shown in Table III. Since we are testing 100 tags of each manufacturer, we decided on averaging the results.

\section{CONClusion}

In this work, we are attempting to identify UHF RFID tags using hidden Markov models. In order to achieve this task, we recorded the transmission of 300 tags from three different manufacturers. In order to build the Markov models, we used five observation streams. The first obser- vation stream is the raw time-voltage waveform data we recorded. The remaining four streams are a processed form of the first time-voltage observation stream. Two main experiment were performed, manufacturer identification and individual tag identification. Regarding the manufacturer identification problem, the experiments showed that it is better to develop models based on timing observations and models based on power observations, and only combine the results based on a weighted equation, since it yielded a TPR of $97 \%$ and an accuracy of $97.67 \%$. As for individual tag identification, we got poor TPR results because only five instances of each tag were acquired. Four out of the five instance were used to build the models and the remaining instance was used to test the model. In the end, when identifying tags, we recommend processing the data we have, and combining the data in some fashion to get the best results.

\section{ACKNOWLEDGMENT}

This material is based upon work supported by the National Science Foundation, CISE/CNS Trustworthy Computing program, under grant No. CNS-1053286.

\section{REFERENCES}

[1] L. R. Rabiner, "A tutorial on hidden markov models and selected applications in speech recognition," A. Waibel and K.-F. Lee, Eds. San Francisco, CA, USA: Morgan Kaufmann Publishers Inc., 1990, ch. Readings in speech recognition, pp. 267-296. [Online]. Available: http://portal.acm.org/citation.cfm?id=108235.108253

[2] S. Chinnappa Gounder Periaswamy, D. R. Thompson, and J. Di, "Fingerprinting RFID tags," IEEE Trans. Dependable and Secure Computing, vol. 8, no. 6, pp. 938 -943, Nov./Dec. 2011.

[3] V. Ltd, http://www.voyantic.com/, 2011.

[4] V. Lakafosis, A. Traille, H. Lee, E. Gebara, M. Tentzeris, G. DeJean, and D. Kirovski, "RFID-CoA: The RFID tags as certificates of authenticity," in IEEE Intl Conf. RFID, Apr. 2011, pp. 207 -214.

[5] D. Zanetti, B. Danev, and S. Capkun, "Physical-layer identification of UHF RFID tags," in Proceedings of the sixteenth annual international conference on Mobile computing and networking, ser MobiCom '10. New York, NY, USA: ACM, 2010, pp. 353-364. [Online]. Available: http://doi.acm.org/10.1145/1859995.1860035

[6] "NEXJEN systems," http://www.nexjen.com/, September 2011.

[7] K. Murphy, Hidden Markov Model (HMM) Toolbox for Matlab, June 2005.

TABLE III: Individual Tag Identification Results using HMM

\begin{tabular}{|c|c|c|c|c|c|c|}
\hline \multirow[b]{2}{*}{ Observations Used } & \multicolumn{2}{|c|}{ Manufacturer A } & \multicolumn{2}{|c|}{ Manufacturer B } & \multicolumn{2}{|c|}{ Manufacturer C } \\
\hline & TPR & Accuracy & TPR & Accuracy & TPR & Accuracy \\
\hline Voltage & $5.00 \%$ & $98.10 \%$ & $19.00 \%$ & $98.38 \%$ & $6.00 \%$ & $98.12 \%$ \\
\hline Power & $8.00 \%$ & $98.16 \%$ & $16.00 \%$ & $98.32 \%$ & $18.00 \%$ & $98.36 \%$ \\
\hline Power and time in the same observations stream & $1.00 \%$ & $98.02 \%$ & $1.00 \%$ & $98.02 \%$ & $1.00 \%$ & $98.02 \%$ \\
\hline Power and Time in separate models & $3.00 \%$ & $96.06 \%$ & $2.00 \%$ & $98.04 \%$ & $1.00 \%$ & $98.02 \%$ \\
\hline
\end{tabular}

